\title{
Kondo Effect in a Few-Electron Quantum Ring
}

\author{
U. F. Keyser, ${ }^{*}$ C. Fühner, S. Borck, and R. J. Haug \\ Institut für Festkörperphysik, Universität Hannover, Appelstrasse 2, 30167 Hannover, Germany
}

M. Bichler and G. Abstreiter

Walter Schottky Institut, TU München, 85748 Garching, Germany

W. Wegscheider

Angewandte und Experimentelle Physik, Universität Regensburg, 93040 Regensburg, Germany

(Received 12 June 2002; published 14 May 2003)

A small quantum ring with less than ten electrons was studied by transport spectroscopy. For strong coupling to the leads a Kondo effect is observed and used to characterize the spin structure of the system in a wide range of magnetic fields. At small magnetic fields Aharonov-Bohm oscillations influenced by Coulomb interaction appear. They exhibit phase jumps by $\pi$ at the Coulomb-blockade resonances. Inside Coulomb-blockade valleys the Aharonov-Bohm oscillations can also be studied due to the finite conductance caused by the Kondo effect. Astonishingly, the maxima of the oscillations show linear shifts with increasing magnetic field and gate voltage.

DOI: 10.1103/PhysRevLett.90.196601

The characterization of semiconductor quantum dots by transport spectroscopy is an extremely successful approach to the understanding of the physics of interacting electrons confined to a quasizero dimensional potential well [1]. Until recently the only accessible shape of these quantum dots was a tiny disc or box with a simple topology. With new fabrication techniques it is now possible to create more complex, multiple connected topologies, namely, small quantum rings with an outer and an inner boundary. These novel devices combine characteristics of classical quantum dots with electronic interference phenomena such as the Aharonov-Bohm (AB) effect [2].

First small quantum rings were fabricated by selfassembled growth of InAs on GaAs [3], but these structures were mainly used for optical experiments. An alternative approach, the local oxidation of GaAs/ AlGaAs heterostructures with an atomic force microscope (AFM) [4], allows one to directly write tuneable quantum rings into a two-dimensional electron gas (2DEG) $[5,6]$. These rings can be studied by tunneling experiments. Because of their small size, transport is dominated by Coulomb blockade (CB) [1] and the number of electrons on such a ring can be controlled by an external gate voltage. Recently, Fuhrer et al. studied a quantum ring containing a few hundred electrons [5]. Their measurements showed an AB effect and allowed one to deduce the energy spectra of their device. It turned out that their system can be well described within a single-particle picture [5] because of an effective screening of the electron-electron interaction by a metallic top gate.

Here we discuss a small quantum ring containing less than ten electrons in a totally different regime. Because of the lack of a screening top gate the ground state of our
PACS numbers: 72.15.Qm, 73.21.La, 73.23.Hk, 73.40.Gk

ring is dominated by strong electron-electron interactions. For a ring in this regime a reduced $A B$ period is predicted [7]. We find indeed such a reduction of the AB period in the transport measurements of our small quantum ring. Furthermore, a strong coupling of the AFMfabricated device to the leads allows us to investigate a Kondo effect [8]. We show that this well-known manybody phenomenon [9-11] is also present in our system. Our measurements prove that electrons confined on our quantum ring form a spin singlet state with electrons in the leads. The finite Kondo conductance allows us to study the $A B$ interference effects even in $C B$ valleys. We report a smooth shift of the AB oscillations with magnetic field and gate voltage and compare it to recent results for a quantum dot embedded into one arm of an $\mathrm{AB}$ ring [12].

We fabricated our quantum ring from a $\delta$-doped $\mathrm{GaAs} / \mathrm{AlGaAs}$-heterostructure containing a 2DEG $34 \mathrm{~nm}$ below the surface. Details on the layer structure can be found in Ref. [6]. The 2DEG has an electron density of $n_{e} \sim 4 \times 10^{15} \mathrm{~m}^{-2}$ and a mobility $\mu_{e} \sim 50 \mathrm{~m}^{2} \mathrm{~V}^{-1} \mathrm{~s}^{-1}$ at low temperatures. A detailed description of our fabrication process was published elsewhere [13]. An AFM image of the completed ring structure is shown in Fig. 1(a). The two in-plane gates $A$ and $B$ are separated from the ring by the rough oxide lines. The ring is connected to the leads by two $150 \mathrm{~nm}$ wide point contacts. Both point contacts are tuned by gate $A$ whereas gate $B$ couples only to the source contact. The inner diameter of the ring is $190 \mathrm{~nm}$ and the outer diameter $450 \mathrm{~nm}$. In the following experiments, gate $A$ is kept at a constant voltage $V_{A}$, and $V_{B}$ is used to control the number of electrons on the ring. Our measurement setup is depicted in Fig. 1(b). All transport measurements were performed in a ${ }^{3} \mathrm{He} /{ }^{4} \mathrm{He}$-dilution refrigerator at a base temperature $T_{b} \sim 30 \mathrm{mK}$ with an ac-excitation voltage of $5 \mu \mathrm{V}$ 


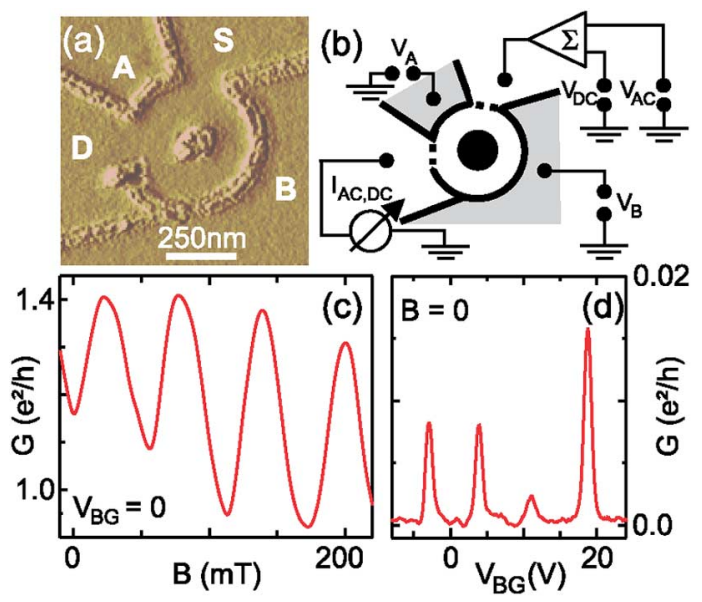

FIG. 1 (color online). (a) AFM image of the quantum ring with an inner diameter of $190 \mathrm{~nm}$ and an outer of $450 \mathrm{~nm}$. (b) Scheme of our measurement setup. (c) Aharonov-Bohm oscillations in the open regime of the quantum ring $\left(V_{A}, V_{B}>\right.$ $0 \mathrm{mV}$ ). (d) Coulomb-blockade oscillations as a function of the backgate voltage $\left(V_{A}=-250 \mathrm{mV}, V_{B}=-100 \mathrm{mV}, B=0\right)$.

at $89 \mathrm{~Hz}$, added to a variable dc voltage $V_{\mathrm{SD}}$. From the temperature dependence of the $\mathrm{CB}$ peaks we deduce an effective temperature of less than $\sim 50 \mathrm{mK}$ for the electronic system.

From the $\mathrm{AB}$ measurements shown in Fig. 1(c) we obtain an $\mathrm{AB}$ period $\Delta B \sim 60 \mathrm{mT}$ at $V_{A}, V_{B} \geq 0 \mathrm{mV}$ for electrons that are transmitted ballistically in a perpendicular magnetic field. The observed periodicity corresponds to a diameter of $300 \mathrm{~nm}$ for the electronic orbit, which fits perfectly to the geometric values. For measurements in the CB regime we apply $V_{A}, V_{B}<-50 \mathrm{mV}$ to separate the ring from the contacts by tunneling barriers. Typical CB oscillations as a function of the voltage $V_{\mathrm{BG}}$ applied to a metallic back gate are shown in Fig. 1(d). By analyzing CB diamonds from nonlinear conductance measurements we extract a charging energy of $U \sim$ $1.5 \mathrm{meV}$ and a single-particle level spacing of $\delta E \sim$ $150 \mu \mathrm{eV}$.

The electron addition spectrum of our small quantum ring is shown in Fig. 2 for magnetic fields up to $B=6 \mathrm{~T}$. The linear conductance $G\left(V_{\mathrm{SD}}=0\right)$ is plotted in gray scale as a function of $V_{B}$ and $B$ at $V_{A}=-80 \mathrm{mV}$. Each CB peak appears as a black line more or less parallel to the $B$ axis. Signatures of nonvanishing conductance between CB peaks are observed and attributed to the Kondo effect, e.g., as marked by the arrow in Fig. 2. If electrons are added according to Hund's rule [14], a Kondo effect is expected in consecutive CB valleys [15]. In contrast, we observe an alternation of the Kondo effect with electron number on the ring (odd-even Kondo effect) below a gate voltage of $-200 \mathrm{mV}$. For $V_{B}>-200 \mathrm{mV}$ a more complicated pattern shows up at small $B$ presumably caused by the opening of the source tunneling barrier and an increased asymmetry of the device.

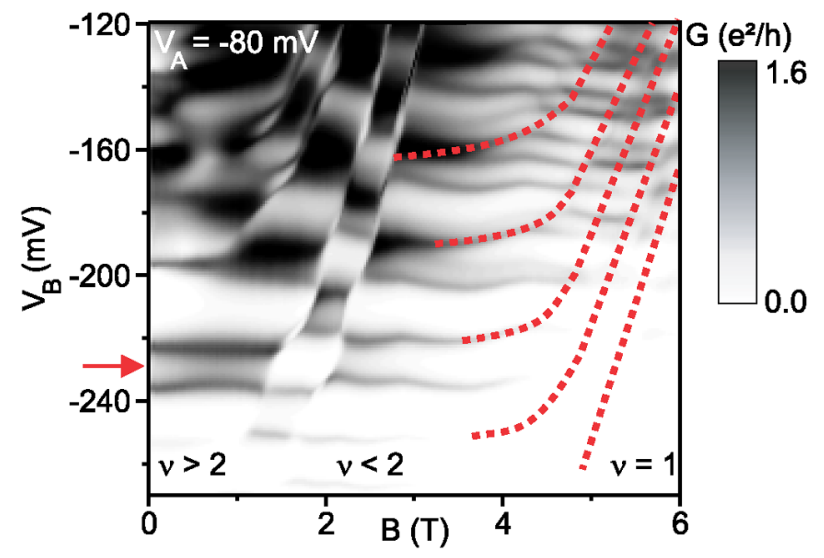

FIG. 2 (color online). Linear conductance $G$ in function of the perpendicular magnetic field $B$ and the gate voltage $V_{B}$.

An alternating pattern of high and low conductance is also observed as a function of $B$. The Kondo effect is modulated abruptly between high (gray) and low (white) conductance regions for $B<2 \mathrm{~T}$. Interestingly, our measurements (Fig. 2) show some similarities with results obtained for quantum dots designed as discs $[15,16]$. This is presumably due to the similar importance of the outer edge for tunneling through quantum dots as well as quantum rings in high magnetic fields. The alternating pattern of the valley conductance with increasing magnetic field can be explained by a redistribution of electrons between different Landau levels (LL) [17]. For example, inside the valley marked in Fig. 2 we observe an increased conductance indicating a Kondo effect with an unpaired spin in the transport state at small $B$. At $B \sim$ $1.5 \mathrm{~T}$ an electron from the upper LL is transferred to the lower LL which is indicated by the sharp boundary in the spectrum. The Kondo effect is suppressed because here the transport level in the lowest and outermost LL $n=0$ contains two electrons with opposite spins $(N=$ odd, spin- 0 in $n=0$ ). At $B \sim 2.0 \mathrm{~T}$ a second electron is transferred to LL $n=0$ and thus an unpaired spin is available again in LL $n=0$. The Kondo effect is restored. For higher $B$ similar drastic changes are not observed anymore. We conclude that no further electrons are redistributed from LL $n=1$ to $n=0$ and therefore assume that all electrons are in the lowest LL (filling factor $\nu=2$ ).

With a further increase of the magnetic field up to $B \sim$ $5.5 \mathrm{~T}$ we observe some weaker features highlighted in Fig. 2 by dashed lines. These small variations in the amplitude and position of the $\mathrm{CB}$ peaks are identified with spin flips of the electrons in the lowest LL. Their spin is flipped from $|\uparrow\rangle$ to $|\downarrow\rangle$ by increasing $B$ [18]. For $B>5.5 \mathrm{~T}$, the electrons in the lowest LL are totally spin polarized corresponding to a filling factor $\nu=1$. The number of electrons $N$ on the ring is determined by counting the spin flips between $\nu=2(B=2 \mathrm{~T})$ and $\nu=1(B \sim 5.5 \mathrm{~T})$. For the marked Kondo valley, we observe only two spin flips. The occurrence of the Kondo 
effect at $B=0$ indicates that $N$ is odd, thus we conclude that there are five electrons on the quantum ring. The same considerations apply for the surrounding CB valleys, for which one extracts $N=4$ and $N=6$, respectively.

To analyze this Kondo effect in more detail, nonlinear transport measurements are shown in Fig. 3. Figure 3(a) depicts the differential conductance $d I_{\mathrm{SD}} / d V_{\mathrm{SD}}$ measured at a temperature of $T_{b}=30 \mathrm{mK}$ as a function of $V_{B}$ and $V_{\mathrm{SD}}\left(V_{A}=-80 \mathrm{mV}, B=0\right)$. The central CB diamond corresponds to the valley discussed above. A sharp zerobias peak appears, whereas in the valleys to the left and right only low conductance is observed. Figure 3(b) depicts temperature dependent measurements at the gate voltage marked in Fig. 3(a). The zero-bias peak observed at $T_{b}=30 \mathrm{mK}$ vanishes almost completely when the temperature is increased to $T \sim 500 \mathrm{mK}$ as expected for a Kondo resonance [19]. From these measurements

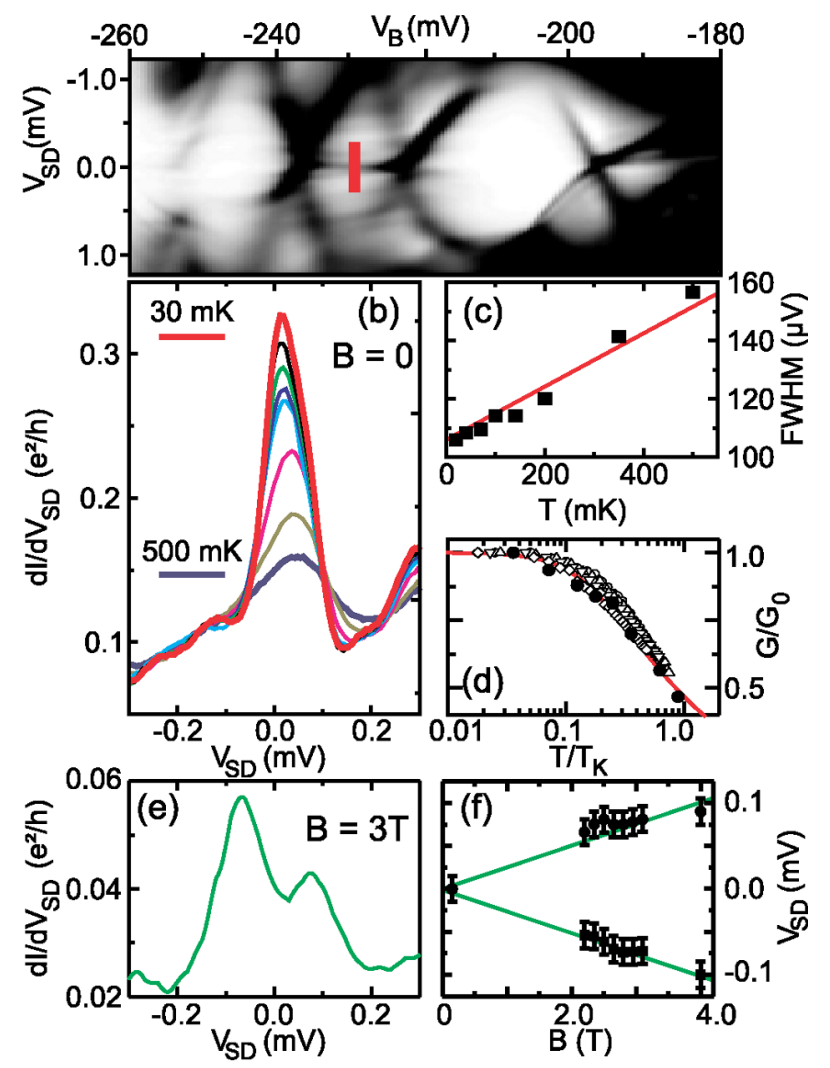

FIG. 3 (color online). (a) Gray scale plot of the differential conductance as a function of $V_{B}$ and source-drain voltage $V_{\mathrm{SD}}$ at $V_{A}=-80 \mathrm{mV}$ and $B=0$ with black (white) high (low) $G$. (b) Temperature dependence of the zero-bias peak in the center of the $N=5$-diamond [marked by the short bar in (a)], $T=30$, 50, 70, 100, 140, 200, 350, $500 \mathrm{mK}$. (c) $T$-dependent full width at half maximum (FWHM). (d) Peak conductance scaled with respective $T_{K}$ and $G_{0}$ (see text) for data in (b) (full circles) and for a stronger tunnel coupling (open symbols). (e) Split Kondo resonance at $B=3$ T. (f) $B$-dependent positions of the split Kondo peaks. we estimate a Kondo temperature $T_{K}$ by extrapolation of the full peak width at half maximum $\Delta V_{\mathrm{SD}}$ to $T=$ $0 \mathrm{~K}$ [Fig. 3(c)], which results in $T_{K} \approx e \Delta V_{\mathrm{SD}, T=0} / 2 k_{B} \sim$ $600 \mathrm{mK}[19,20]$. Fitting the Kondo conductance at zero-bias $G(T)$ with an empirical formula from Refs. $\quad[20-22], \quad G(T)=G_{0} /\left[1+\left(2^{1 / s}-1\right)\left(T / T_{K}\right)^{2}\right]^{s}$ yields $T_{K} \sim 600 \mathrm{mK}$ and $s \sim 0.21$. This is in agreement with earlier studies on a spin-1/2 system [22]. The scaled peak conductance (full circles) is shown together with the fit in Fig. 3(d). To prove the scaling behavior, results obtained at four different gate voltages are shown as open symbols in Fig. 3(d). The respective Kondo temperatures are $T_{K} \sim 0.9,1.0,1.1,1.4 \mathrm{~K}$.

The zero-bias peak of a spin-1/2 Kondo effect is expected to split with increasing magnetic field according to the Zeeman effect, $\Delta E=g_{\mathrm{GaAs}} \mu_{B} B\left(g_{\mathrm{GaAs}}=0.44, \mu_{B}\right.$ Bohr's magneton). This peak splitting is shown at $B=$ $3 \mathrm{~T}$ in Fig. 3(e). The different peak amplitudes are related to a slight asymmetry in the coupling of the ring to the leads. The peak positions in $V_{\mathrm{SD}}$ extracted from several measurements for $2 \mathrm{~T}<B<4 \mathrm{~T}$ are plotted in Fig. 3(f), the lines indicate the expected peak positions. We observe a nice agreement with the measurement for $B>2 \mathrm{~T}$ which is another evidence for a spin-1/2 Kondo effect. Between 1.5 and $2.1 \mathrm{~T}$ the Kondo effect is absent due to the paired spin configuration. For $B<1.5 \mathrm{~T}$ the peak splitting is not resolved, presumably due to the broadening of the Kondo peak of the order of $k_{B} T_{K} \sim 50 \mu \mathrm{eV}$.

The CB peaks are broadened due to the strong coupling in the Kondo regime as well [dashed line in Fig. 4(a), $\left.V_{A}=-80 \mathrm{mV}\right]$. This broadening obscures the small shifts in the peak positions as induced by changes of the ground states in the ring. To avoid this, the Kondo valley marked in Fig. 2 is shown again in Fig. 4(a) at a slightly lower tunnel coupling at $V_{A}=-150 \mathrm{mV}$. This reduces the conductance in the Kondo regime to below $0.1 e^{2} / h$. Because of the finite capacitance between the ring and gate $A$, the center of the valley is shifted from $V_{B} \sim-230 \mathrm{mV}$ to $V_{B} \sim-185 \mathrm{mV}$. In Fig. 4(b) we show AB oscillations in the normalized conductance $G / G_{0}$ as a function of $B$ for the gate voltages marked by the symbols in Fig. 4(a). The vertical dashed lines denote the expected period of $\Delta B \sim 60 \mathrm{mT}$ of the AB oscillations extracted from the measurements in the open regime. It is immediately evident that we obtain a much shorter period. This shorter period is in contrast to the results of Fuhrer et al. [5], who obtained the normal AB period for their ring with many electrons and screened electron-electron interaction. A comparable oscillation period is also obtained in the center of the Kondo valley at higher tunnel coupling $V_{A}=-80 \mathrm{mV}$ and $V_{B}=-231 \mathrm{mV}$ [marked by the open circle in (a)] as depicted in the lower part of Fig. 4(b). The fast AB oscillations are also reflected by the movement of the CB peaks in Fig. 4(c) as well. Each kink in the $\mathrm{CB}$ peak position indicates a change of the ground state in our ring. 


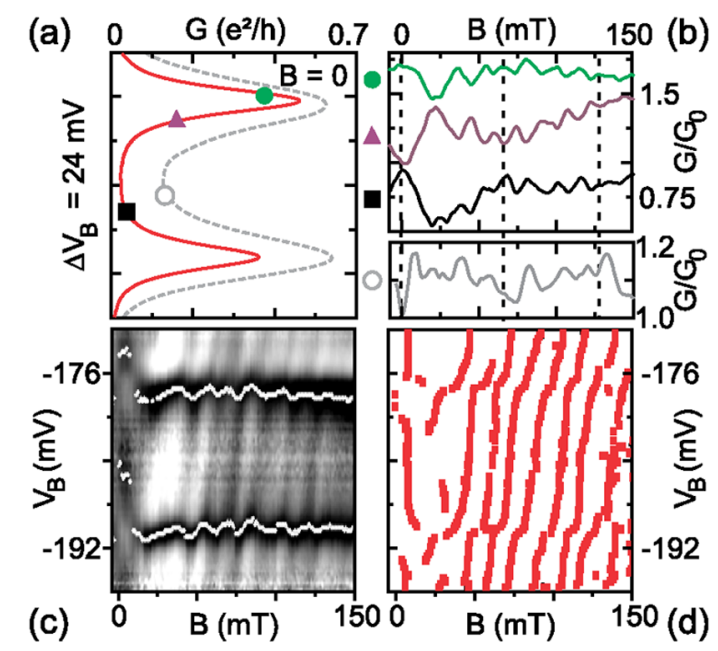

FIG. 4 (color online). (a) $N=5$-Kondo valley at $V_{A}=$ $-150 \mathrm{mV}$ (solid) and $V_{A}=-80 \mathrm{mV}$ (dashed line) at $B=0$. $\boldsymbol{\square}, \boldsymbol{\Delta}, \boldsymbol{O}$, and $\bigcirc$ mark the gate voltages for the AharonovBohm measurements in (b). (b) Upper part: Normalized conductance $G / G_{0}$ as a function of $B$ at $V_{A}=-150 \mathrm{mV}$. The curves are offset for clarity. Lower part: $G / G_{0}$ at $V_{A}=$ $-80 \mathrm{mV}$ after subtraction of an increasing background. (c) $G / G_{0}\left(B, V_{B}\right)$ as gray scale plot at $V_{A}=-150 \mathrm{mV}$ with peaks marked by white dots. (d) Positions of the AharonovBohm maxima extracted from (c).

According to a theoretical calculation of Niemelä et al. electron-electron interaction lifts the degeneracy of the singlet and triplet states in a ring [7]. This effect leads to more frequent level crossings of the ground state compared to a ring without interaction because the triplet states are present at lower energies. These calculations predict a transition $\mathrm{AB}$ period $\Delta B$ that shortens with increasing number of electrons on the ring, e.g., for four electrons it should be 4 times smaller. For our ring with five electrons $\Delta B$ should be shortened by a factor of 5 . In fair agreement, we observe a period of $\Delta B \sim 13 \mathrm{mT}$ which is 4 to 5 times shorter compared to the open regime. The deviations from perfect periodicity especially at small magnetic fields have to be explained by the influence of some residual disorder.

We utilize the Kondo effect with its finite conductance to study the evolution of the AB effect in the CB valley. In Fig. 4(d) each square marks the position of an AB maximum in the $\left(V_{B}, B\right)$ plane extracted from the data presented in Fig. 4(c). The small kinks, visible for all magnetic fields at both CB peaks at $V_{B} \sim-190 \mathrm{mV}$ and $V_{B} \sim-177 \mathrm{mV}$ indicate a phase jump of $\pi$. This result is verified in Fig. 4(b): the conductance at $B=0$ changes

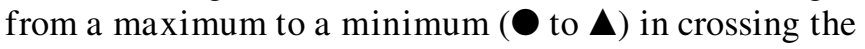
peak. Apparently, we observe smooth linear shifts of the AB maxima in Fig. 4(d), indicated by the slightly tilted vertical lines. These appear in our two-terminal measurement only at finite magnetic field when the ring is threaded by at least half a flux quantum. Linear shifts of normal $\mathrm{AB}$ oscillations were recently reported by $\mathrm{Ji}$ et al. for a quantum dot in the unitary Kondo regime embedded in an $\mathrm{AB}$ interferometer [12]. Their fourterminal measurement is interpreted in terms of smooth phase shifts by almost $2 \pi$ across a Kondo resonance. In contrast we investigate a Kondo resonance far from the unitary limit in a quantum dot which itself serves as the interferometer. The exact mechanism for the observed linear shift of the AB maxima is still to be clarified, but it might be connected to the fact that the level structure of our small ring interferometer is influenced by the gate voltage. For a detailed understanding further theoretical work is necessary.

In conclusion, a small tuneable quantum ring with less than ten electrons is shown to exhibit AharonovBohm oscillations as well as Coulomb blockade. At strong coupling to the leads we find evidence of a Kondo effect induced by a single spin on the ring. The energy spectrum is strongly influenced by electronelectron interaction. An analysis of the phase evolution of the Aharonov-Bohm effect in the Kondo regime yields phase jumps by $\pi$ at the Coulomb-blockade resonances and a smooth shift of the Aharonov-Bohm maxima in between.

We acknowledge discussions with S. Ulloa and J. König and financial support by BMBF, DIP, and TMR.

*Email address: keyser@mb.tn.tudelft.nl

[1] L. P. Kouwenhoven et al., in Mesoscopic Electron Transport, edited by L. L. Sohn, L. P. Kouwenhoven, and G. Schön (Kluwer, Dordrecht, 1997), pp. 105-214.

[2] Y. Aharonov and D. Bohm, Phys. Rev. 115, 485 (1959).

[3] A. Lorke et al., Phys. Rev. Lett. 84, 2223 (2000).

[4] M. Ishii and K. Matsumoto, Jpn. J. Appl. Phys. 34, 1329 (1995).

[5] A. Fuhrer et al., Nature (London) 413, 822 (2001).

[6] U. F. Keyser et al., Semicond. Sci. Technol. 17, L22 (2002).

[7] K. Niemelä et al., Europhys. Lett. 36, 533 (1996).

[8] J. Kondo, Prog. Theor. Phys. 32, 37 (1964).

[9] T. K. Ng and P. A. Lee, Phys. Rev. Lett. 61, 1768 (1988).

[10] L. I. Glazman et al., JETP Lett. 47, 452 (1988).

[11] D. Goldhaber-Gordon et al., Nature (London) 391, 156 (1998); S. M. Cronenwett et al., Science 281, 540 (1998); J. Schmid et al., Physica (Amsterdam) 256B, 182 (1998).

[12] Y. Ji et al., Phys. Rev. Lett. 88, 076601 (2002).

[13] U. F. Keyser et al., Appl. Phys. Lett. 76, 457 (2000).

[14] S. Tarucha et al., Phys. Rev. Lett. 77, 3613 (1996).

[15] J. Schmid et al., Phys. Rev. Lett. 84, 5824 (2000).

[16] D. Sprinzak et al., Phys. Rev. Lett. 88, 176805 (2002).

[17] P. L. McEuen et al., Phys. Rev. B 45, 11419 (1992).

[18] M. Ciorga et al., Phys. Rev. B 61, R16315 (2000).

[19] Y. Meir et al., Phys. Rev. Lett. 70, 2601 (1993).

[20] J. Nygard et al., Nature (London) 408, 342 (2000).

[21] T. A. Costi et al., J. Phys. Condens. Matter 6, 2519 (1994).

[22] D. Goldhaber-Gordon et al., Phys. Rev. Lett. 81, 5225 (1998). 